\title{
Preoperative Magnetic Resonance Imaging Evaluation in Patients with Adolescent Idiopathic Scoliosis
}

\author{
Choon Sung Lee, Chang Ju Hwang, Nam Heun Kim, \\ Hyun Min Noh, Mi Young Lee, So Jung Yoon, Dong-Ho Lee
}

Scoliosis Center, Department of Orthopaedic Surgery, Asan Medical Center, University of Ulsan College of Medicine, Seoul, Korea

\begin{abstract}
Study Design: Retrospective case series.
Purpose: The purpose of this study was to examine the incidence of neural axis abnormalities and the relevant risk factors in patients with adolescent idiopathic scoliosis (AIS).

Overview of Literature: The use of preoperative magnetic resonance imaging (MRI) to assess the whole spine in patients with idiopathic scoliosis is controversial, and indications for such MRI evaluations have not been definitively established. However, we routinely use whole-spine $\mathrm{MRI}$ in patients with scoliosis who are scheduled to undergo surgical correction.

Methods: A total of 378 consecutive patients with presumed AIS who were admitted for spinal surgery were examined for neural axis abnormalities using MRI. To differentiate patients with normal and abnormal MRI findings, the following clinical parameters were evaluated: age, sex, menarcheal status, rotation angle (using a scoliometer), coronal balance, shoulder height difference, and low back pain. We radiographically evaluated curve type, thoracic or thoracolumbar curve direction, curve magnitude and flexibility, apical vertebral rotation, curve length, coronal balance, sagittal balance, shoulder height difference, thoracic kyphosis, and the Risser sign.

Results: Neural axis abnormalities were detected in 24 patients (6.3\%). Abnormal MRI findings were significantly more common in males than in females and were associated with increased thoracic kyphosis. However, there were no significant differences in terms of the other measured parameters.

Conclusions: Among the patients with presumed AIS who received preoperative whole-spine MRI, $6.3 \%$ had neural axis abnormalities. Males and patients with increased thoracic kyphosis were at a higher risk.
\end{abstract}

Keywords: Neural axis abnormality; Magnetic resonance imaging; Adolescent idiopathic scoliosis

\section{Introduction}

Idiopathic scoliosis is diagnosed by the exclusion of other etiologies that cause spinal deformities, such as congenital deformities, neuromuscular disorders, various genetic syndromes, and tumors. However, neural axis abnormali- ties may be found in patients who have atypical clinical features of scoliosis without any specific etiology. Though the incidence of neural axis abnormalities has been shown to be high in young patients and in patients with a left thoracic curve [1-6], the associated clinical and radiological characteristics have not been clearly elucidated.

\footnotetext{
Received Apr 9, 2016; Revised Jul 6, 2016; Accepted Jul 28, 2016

Corresponding author: Chang Ju Hwang

Scoliosis Center, Department of Orthopaedic Surgery, Asan Medical Center,

88 Olympic-ro 43-gil, Songpa-gu, Seoul 05505, Korea

Tel: +82-2-3010-3536, Fax: +82-2-488-7877, E-mail: baski47@gmail.com
} 
Therefore, examining patients with adolescent idiopathic scoliosis (AIS) using spinal magnetic resonance imaging (MRI) remains controversial.

Neural axis abnormalities are found in neurologically normal patients and are clinically relevant when planning surgical treatment for scoliosis. It is well known that there may be a risk of neurological complications when scoliosis corrections are performed without decompression in patients with syringomyelia, the most common neural axis abnormality [7-9]. Therefore, determining the presence of neural axis abnormalities using preoperative wholespine MRI can be critical. While some surgeons claim that decompression should be performed on all patients with syringomyelia before surgical correction [7-9], others suggest that MRI and decompression are not necessary in patients with presumed idiopathic scoliosis without associated neurological abnormalities [2,10-15].

Such controversies are attributed to the fact that indications for MRI in patients with idiopathic scoliosis vary, and the exact prevalence of neural axis abnormalities has not been established. However, we can perform preoperative whole-spine MRI on all patients with presumed idiopathic scoliosis who are scheduled to undergo surgical correction. The purpose of this study was to use MRI examinations to determine the incidence of neural axis abnormalities and relevant clinical and radiological risk factors.

\section{Materials and Methods}

A total of 374 consecutive patients with presumed AIS for whom surgical treatment was planned between 2005 and 2011 were included. Whole-spine MRI was performed on all patients, and there were no missing data sets. Patients with congenital anomalies, neuromuscular disorders, neurofibromatosis, preexisting neural axis abnormalities, or syndromes known to be associated with spinal deformities were excluded. No patients included in this study had evidence of non-idiopathic scoliosis.

The medical records of all patients were reviewed to determine the child's age at presentation, sex, menarcheal status, angle of trunk rotation (as measured on a scoliometer), coronal balance, shoulder height difference, neurological abnormalities, and presence of back pain. Radiological parameters, including curve type, direction of the thoracic/thoracolumbar curve, magnitude, length and flexibility of the main curve, rotation of the apical vertebra, coronal and sagittal balance, shoulder height dif- ference, thoracic kyphosis, lumbar lordosis, and the Risser grade were measured on whole-spine standing radiographs and on supine side-bending radiographs obtained while assistants maximally straightened the curve. All parameters were measured immediately before surgery.

Coronal balance was clinically and radiographically classified as $<10 \mathrm{~mm}, 10$ to $<20 \mathrm{~mm}, 20$ to $<30 \mathrm{~mm}$, or $\geq 30 \mathrm{~mm}$. Shoulder height difference was clinically and radiographically classified as $<10 \mathrm{~mm}$, or $\geq 10 \mathrm{~mm}$. If the measured value was exactly 10 or 20 or 30 , it was included in the group with larger values. The thoracic sagittal profile was not included in the analysis based on the Lenke classification system. Apical vertebral rotation was graded using the Nash-Moe method (grade 0-4). Sagittal balance was expressed in millimeters by measuring the distance between the $\mathrm{C} 7$ plumb line and the posterosuperior corner of S1 vertebrae. Thoracic kyphosis was measured from the superior endplate of $\mathrm{T} 5$ to the inferior endplate of T12 using the Cobb method, and lumbar lordosis was measured from the superior endplate of L1 to the superior endplate of S1.

The position of the conus medullaris, the presence or absence of syringomyelia, Chiari malformations, and tonsillar ectopia were noted, and corresponding treatment was described. The abovementioned clinical and radiographic parameters of patients with normal MRI findings were compared with those of patients with neural axis abnormalities. The chi-square or Fisher exact test was used to assess categorical variables, and Student's $t$ test or the Kruskal-Wallis test was used to assess continuous variables.

\section{Results}

Abnormal neural axis findings were observed on the whole-spine MRI findings in 24 of the 378 patients (6.3\%). Most neural axis abnormalities were syringomyelia with 20 cases of syringomyelia and 5 of Chiari malformation. Two patients had both syringomyelia and Chiari malformation. Dural ectasia due to an unknown cause was identified in another patient. All patients with abnormal findings were referred to a neurosurgeon for further evaluation. Eighteen patients with syringomyelia only received follow-up examinations after deformity correction without any treatment for syringomyelia. The three patients with Chiari malformation only underwent foramen magnum decompression and proceeded to surgical 
Table 1. Abnormal neural axis findings observed on the whole-spine MRIs

\begin{tabular}{lcc} 
Variable & No. of patients & Neurosurgical intervention \\
Syrinx only & 18 & 0 \\
Chiari malformation only & 3 & 1 \\
Syrinx and Chiari malformation & 2 & 2 \\
Other & 1 & 0 \\
\hline Total & 24 & 3 \\
\hline
\end{tabular}

$\mathrm{MRI}$, magnetic resonance imaging.

Table 2. Clinical parameters and MRI abnormalities

\begin{tabular}{|c|c|c|c|}
\hline Variable & Normal group & Abnormal group & $p$-value \\
\hline Age (yr) & 14.7 & 15.8 & 0.786 \\
\hline Male (\%) & 17.5 & 37.5 & 0.027 \\
\hline Premenarche (\%) & 23.3 & 6.7 & 0.243 \\
\hline Scoliometer $\left({ }^{\circ}\right)$ & 12.7 & 13.8 & 0.159 \\
\hline Coronal balance (\%) & & & 0.253 \\
\hline$<10 \mathrm{~mm}$ & 48.9 & 51.7 & \\
\hline $10-20 \mathrm{~mm}$ & 35.9 & 24.1 & \\
\hline $20-30 \mathrm{~mm}$ & 10.1 & 20.7 & \\
\hline$>30 \mathrm{~mm}$ & 5.2 & 3.5 & \\
\hline Shoulder-height difference (<10 mm, \%) & 50.4 & 51.7 & 0.894 \\
\hline Neurological abnormalities (normal, \%) & 98.6 & 95.8 & 0.327 \\
\hline Back pain (\%) & 20.6 & 8.3 & 0.189 \\
\hline
\end{tabular}

MRI, magnetic resonance imaging.

deformity correction (Table 1).

The male-to-female ratio was significantly higher among patients with neural axis abnormalities than among those with normal findings $(p=0.027)$. The thoracic kyphosis angle was significantly increased among patients with neural axis abnormalities than among those with normal findings $\left(21.9^{\circ}\right.$ vs. $\left.16.3^{\circ}, p=0.011\right)$. No statistically significant differences were observed between patients with normal and those with abnormal MRI findings in terms of the other parameters measured. Five patients with normal MRI findings and one patient with neural axis abnormalities had neurological abnormalities. Among the five patients with normal MRI findings, three had hyperactive deep tendon reflex (DTR) and two had decreased motor power of the big toe extensor muscle. The patient with neural axis abnormalities had hyperactive DTR. The proportion of patients with a left thoracic or thoracolumbar curve pattern, which is known to be associated with neural axis abnormalities, was higher among patients with normal MRI findings (32 patients, 9.0\%) than among those with neural axis abnormalities (1 patient, 4.2\%) (Tables 2, 3).

Intraoperative neuromonitoring using motor and/or somatosensory evoked potentials (MEP/SSEP) was performed in all patients during correction surgery. Substantial abnormalities in MEP/SSEP data were detected in 29 patients with normal MRI findings (8.2\%) and 3 patients with neural axis abnormalities (12.5\%), with no significant differences between the groups $(p=0.300)$. Although the abnormalities persisted until the end of surgery in some patients, no patient demonstrated postoperative neurological deterioration compared with the preoperative status.

\section{Discussion}

The need to perform routine, preoperative, whole-spine 
Table 3. Radiological parameters and MRI abnormalities

\begin{tabular}{|c|c|c|c|}
\hline Variable & Normal group & Abnormal group & $p$-value \\
\hline Lenke classification (IOR) & 5.0 & 5.5 & 0.773 \\
\hline King classification (\%) & & & 0.831 \\
\hline Type 1 & 22.9 & 13.8 & \\
\hline Type 2 & 31.3 & 34.5 & \\
\hline Type 3 & 22.6 & 27.6 & \\
\hline Type 4 & 7.1 & 10.3 & \\
\hline Type 5 & 12.3 & 13.8 & \\
\hline Curve direction (left \%) & 9.0 & 4.2 & 0.350 \\
\hline Curve magnitude $\left({ }^{\circ}\right)$ & 56.5 & 57.2 & 0.410 \\
\hline Curve length (IOR) & 7.0 & 7.0 & 0.634 \\
\hline Flexibility (\%) & 50.8 & 50.9 & 0.962 \\
\hline Apical vertebral rotation (\%) & & & 0.565 \\
\hline Grade 0 & 3.3 & 3.1 & \\
\hline Grade 1 & 26.2 & 40.6 & \\
\hline Grade 2 & 59.1 & 43.8 & \\
\hline Grade 3 & 8.5 & 9.4 & \\
\hline Grade 4 & 3.0 & 3.1 & \\
\hline Shoulder-height difference (<10 mm, \%) & 39.7 & 38.5 & 0.903 \\
\hline Risser grade (\%) & & & 0.797 \\
\hline Grade 0 & 10.6 & 3.1 & \\
\hline Grade 1 & 7.9 & 15.6 & \\
\hline Grade 2 & 9.5 & 12.5 & \\
\hline Grade 3 & 16.3 & 15.6 & \\
\hline Grade 4 & 28.3 & 21.9 & \\
\hline Grade 5 & 27.5 & 31.3 & \\
\hline Coronal balance (\%) & & & 0.055 \\
\hline$<10 \mathrm{~mm}$ & 48.4 & 54.8 & \\
\hline $10-20 \mathrm{~mm}$ & 36.1 & 19.4 & \\
\hline $20-30 \mathrm{~mm}$ & 11.7 & 25.8 & \\
\hline$>30 \mathrm{~mm}$ & 3.8 & 0 & \\
\hline Sagittal balance (mm) & -1.1 & -11.3 & 0.903 \\
\hline Thoracic kyphosis $\left({ }^{\circ}\right)$ & 16.3 & 21.9 & 0.011 \\
\hline Lumbar lordosis $\left({ }^{\circ}\right)$ & -51.8 & -50.7 & 0.658 \\
\hline
\end{tabular}

$\mathrm{MRI}$, magnetic resonance imaging; IQR, interquartile range.

MRI evaluations in patients with presumed idiopathic scoliosis remains controversial. The incidence of neural axis abnormalities among patients with "idiopathic" scoliosis reported in the literature covers a range from $1.8 \%$ to $18 \%[1,2,5,6,10,11,14,16-20]$. However, the actual incidence remains unknown because few studies have been conducted on a large number of consecutive patients, inclusion criteria vary between studies, and indications for whole-spine MRI differ among surgeons. In addition, there is currently no consensus regarding clinical and radiological risk factors associated with neural axis abnormalities.

This study had several limitations, mostly due to the small sample size and the retrospective design. A study 
by Diab et al. [2] included 2,206 patients and is the largest to date examining the use and outcomes of preoperative spinal MRI to assess AIS. Although it is a prospective, consecutive, and large-scale clinical series, only $41.8 \%$ of the enrolled patients (923 patients) received spinal MRI, but indications for preoperative MRI evaluation were not described. As many as 50 surgeons participated in that study, and the decision to perform MRI on a patient was likely made by the individual surgeon at each center. Although the number of patients was far greater in the study conducted by Diab et al. [2], our study may have an advantage due to the relatively higher homogeneity of the patient population, which comprised consecutive patients undergoing whole-spine MRI at a single center.

A left thoracic curve pattern is one of the most commonly mentioned risk factors for neural axis abnormalities $[3,6,11,16]$. Rothman and Simeone [21] have stated that as most thoracic curves are right-sided, a left thoracic curve is evaluated as another etiology. Wu et al. [6] reported neural axis abnormalities in 37 patients (54\%) from a total of 68 consecutive patients with presumed idiopathic left thoracic scoliosis and suggested that MRI should be considered in patients, particularly males, when a left thoracic curve pattern is present. In our current study, 33 patients had left thoracic or thoracolumbar curves. From these, only one had a neural axis abnormality determined by whole-spine MRI, which is contrary to widely held clinical beliefs. However, in the aforementioned multicenter study by Diab et al. [2], an apex left thoracic curve was not an independent predictor of spinal abnormalities detected by MRI. In our current study, the two statistically significant risk factors were male sex and thoracic kyphosis. Goldberg et al. [3] suggested that male sex and age at onset in females are more important factors than a left curve pattern. According to several recent studies, normal or increased thoracic kyphosis is associated with neural axis abnormalities $[2,16,19,22,23]$. A study by Davids et al. [16] suggested that patients with presumed AIS have lesions in the central nervous system when the sagittal thoracic alignment is $>20^{\circ}$ of kyphosis. In a study by Richards et al. [19], which included 529 patients with Lenke 1-4 right thoracic curve patterns, patients with abnormal MRI findings demonstrated significantly increased thoracic kyphosis on radiography than those with normal MRI findings.

The incidence of neural axis abnormalities was $6.3 \%$ in our study, which is within the range reported by others
$[1,2,5,6,10,11,14,16-20]$. It is noteworthy that no patient in our study had postoperative neurologic deficits, even though neurosurgical interventions were not performed in most patients having neural axis abnormalities. However, we cannot exclude the possibility of neurological issues in the three patients who underwent foramen magnum decompression before surgical deformity correction. Debates regarding the necessity of routine preoperative MRI screening require the determination of the incidence of neural axis abnormalities and whether surgical correction is frequently accompanied by neurologic events in these patients. Some authors have questioned the use of routine MRI evaluations for patients with presumed AIS as the incidence of neural axis abnormalities is low $[2,10-12,14]$, and the study by Diab et al. [2] concluded that there were no differences in complication rates between patients with normal and those abnormal MRI findings, thereby inferring that routine MRI is not needed. On the contrary, authors who advocate routine MRI are concerned that neural axis abnormalities are risk factors for neurologic complications following surgical correction [17,20,22,24]. Singhal et al. [20] suggested that the presence of clinical signs is a poor predictive indicator of neurological abnormalities and that the routine use of MRI should be recommended for all patients who will receive surgical intervention for idiopathic scoliosis.

It is difficult to draw conclusions from the results of our present study regarding the need to perform routine preoperative spinal MRI for patients with presumed AIS. Our results may be more reliable than those of previous studies as we included a relatively large number of consecutive patients who received MRI examinations before surgery. Although the incidence of neural axis abnormalities in our cohort was low and no neurologic deficits developed in our patients, we recommend the routine use of preoperative whole-spine MRI because neurologic complications can lead to irreparable and serious results. These complications can be prevented by administering appropriate neurosurgical interventions before corrective surgery, and the awareness of such abnormalities in advance typically makes the surgeon pay closer attention and perform more meticulous corrective maneuvers.

\section{Conclusions}

Among the patients with presumed AIS who received preoperative whole-spine MRI, $6.3 \%$ had neural axis abnor- 
malities. The main risk factors for neural axis abnormalities were male sex and increased thoracic kyphosis.

\section{Conflict of Interest}

No potential conflict of interest relevant to this article was reported.

\section{References}

1. Benli IT, Uzumcugil O, Aydin E, Ates B, Gurses L, Hekimoglu B. Magnetic resonance imaging abnormalities of neural axis in Lenke type 1 idiopathic scoliosis. Spine (Phila Pa 1976) 2006;31:1828-33.

2. Diab M, Landman Z, Lubicky J, Dormans J, Erickson M, Richards BS. Use and outcome of MRI in the surgical treatment of adolescent idiopathic scoliosis. Spine (Phila Pa 1976) 2011;36:667-71.

3. Goldberg CJ, Moore DP, Fogarty EE, Dowling FE. Left thoracic curve patterns and their association with disease. Spine (Phila Pa 1976) 1999;24:1228-33.

4. Maenza RA. Juvenile and adolescent idiopathic scoliosis: magnetic resonance imaging evaluation and clinical indications. J Pediatr Orthop B 2003;12:295302.

5. Pahys JM, Samdani AF, Betz RR. Intraspinal anomalies in infantile idiopathic scoliosis: prevalence and role of magnetic resonance imaging. Spine (Phila Pa 1976) 2009;34:E434-8.

6. Wu L, Qiu Y, Wang B, Zhu ZZ, Ma WW. The left thoracic curve pattern: a strong predictor for neural axis abnormalities in patients with "idiopathic" scoliosis. Spine (Phila Pa 1976) 2010;35:182-5.

7. Akhtar OH, Rowe DE. Syringomyelia-associated scoliosis with and without the Chiari I malformation. J Am Acad Orthop Surg 2008;16:407-17.

8. Kontio K, Davidson D, Letts M. Management of scoliosis and syringomyelia in children. J Pediatr Orthop 2002;22:771-9.

9. Noordeen MH, Taylor BA, Edgar MA. Syringomyelia: a potential risk factor in scoliosis surgery. Spine (Phila Pa 1976) 1994;19:1406-9.

10. Do T, Fras C, Burke S, Widmann RF, Rawlins B, Boachie-Adjei O. Clinical value of routine preoperative magnetic resonance imaging in adolescent idiopathic scoliosis: a prospective study of three hundred and twenty-seven patients. J Bone Joint Surg Am
2001;83:577-9.

11. Inoue M, Minami S, Nakata Y, et al. Preoperative MRI analysis of patients with idiopathic scoliosis: a prospective study. Spine (Phila Pa 1976) 2005;30:10814.

12. Morcuende JA, Dolan LA, Vazquez JD, Jirasirakul A, Weinstein SL. A prognostic model for the presence of neurogenic lesions in atypical idiopathic scoliosis. Spine (Phila Pa 1976) 2004;29:51-8.

13. Wang G, Sun J, Jiang Z, Cui X, Cui J. One-stage correction surgery of scoliosis associated with syringomyelia: is it safe to leave untreated a syrinx without neurological symptom? J Spinal Disord Tech 2015;28:E260-4.

14. Winter RB, Lonstein JE, Heithoff KB, Kirkham JA. Magnetic resonance imaging evaluation of the adolescent patient with idiopathic scoliosis before spinal instrumentation and fusion: a prospective, doubleblinded study of 140 patients. Spine (Phila Pa 1976) 1997;22:855-8.

15. Zhang ZX, Feng DX, Li P, et al. Surgical treatment of scoliosis associated with syringomyelia with no or minor neurologic symptom. Eur Spine J 2015;24:1555-9.

16. Davids JR, Chamberlin E, Blackhurst DW. Indications for magnetic resonance imaging in presumed adolescent idiopathic scoliosis. J Bone Joint Surg Am 2004;86:2187-95.

17. Hausmann ON, Boni T, Pfirrmann CW, Curt A, Min K. Preoperative radiological and electrophysiological evaluation in 100 adolescent idiopathic scoliosis patients. Eur Spine J 2003;12:501-6.

18. Ozturk C, Karadereler S, Ornek I, Enercan M, Ganiyusufoglu K, Hamzaoglu A. The role of routine magnetic resonance imaging in the preoperative evaluation of adolescent idiopathic scoliosis. Int Orthop 2010;34:543-6.

19. Richards BS, Sucato DJ, Johnston CE, et al. Right thoracic curves in presumed adolescent idiopathic scoliosis: which clinical and radiographic findings correlate with a preoperative abnormal magnetic resonance image? Spine (Phila Pa 1976) 2010;35:185560.

20. Singhal R, Perry DC, Prasad S, Davidson NT, Bruce $\mathrm{CE}$. The use of routine preoperative magnetic resonance imaging in identifying intraspinal anomalies in patients with idiopathic scoliosis: a 10-year review. 
Eur Spine J 2013;22:355-9.

21. Rothman RH, Simeone FA. The spine. 3rd ed. Philadelphia: Saunders Elsevier; 1992.

22. Ferguson RL, DeVine J, Stasikelis P, Caskey P, Allen BL Jr. Outcomes in surgical treatment of "idiopathiclike" scoliosis associated with syringomyelia. J Spinal Disord Tech 2002;15:301-6.

23. Ouellet JA, LaPlaza J, Erickson MA, Birch JG, Burke
S, Browne R. Sagittal plane deformity in the thoracic spine: a clue to the presence of syringomyelia as a cause of scoliosis. Spine (Phila Pa 1976) 2003;28: 2147-51.

24. Lee RS, Reed DW, Saifuddin A. The correlation between coronal balance and neuroaxial abnormalities detected on MRI in adolescent idiopathic scoliosis. Eur Spine J 2012;21:1106-10. 